\title{
Reinforcement and the organization of behavior in golden hamsters: Sunflower seed and nest paper reinforcers
}

\author{
SARA J. SHETTLEWORTH \\ University of Toronto, Toronto, Canada M5S IAI
}

\begin{abstract}
Undeprived hamsters received nest paper or sunflower seeds in their home cages, free or contingent on one of three activities. The pattern of effects of reinforcement was the same as that reported previously with food for hungry hamsters in an open field: Open rearing and scrabbling quickly increased in rate when reinforced with paper or seeds, but face washing showed little or no increase. Certain interrelations found previously between noncontingent effects of reinforcers, deprivation effects, and contingent effects were absent and therefore are not essential to the differences among these three behaviors during reinforcement. The hamsters could learn to interrupt fact washing to collect either paper or seeds, but even then they did not increase their time face washing to high levels. Raising the operant level of face washing by spraying the animals with water did not improve instrumental performance. Face washing could be classified as an involuntary activity, but this term does not provide a full account of its special properties, such as shortened bouts, during reinforcement and punishment.
\end{abstract}

There is much evidence that the effects of instrumental conditioning procedures may be influenced by the particular combination of response and reinforcer used (Shettleworth, 1973; Stevenson-Hinde, 1973). The questions remain whether a general performance rule for instrumental conditioning can be formulated that takes such effects into account and what kinds of motivational and associative mechanisms are implied. Of particular theoretical interest is the question whether apparent failures of reinforcement represent associative "constraints on learning" (Seligman, 1970; Shettleworth, 1972).

The experiments reported here are part of a program to map out the effects of a variety of reinforcers on a variety of responses in one species, the golden hamster, and to analyze the mechanism of any differences among responses and reinforcers that are found. In experiments reported previously (Shettleworth, 1973, 1975), food-deprived hamsters were reinforced with food in a relatively novel environment for performing one of several topographically defined responses, or action patterns (APs). Some increased (digging, open rearing, scrabbling, barpressing), while others showed little or no change (face washing, scratching the

I thank Michael R. Juergensen and Cheryl Rosenblum-Blinick for help in conducting the experiments and William R. Bullock for his technical assistance. BA thesis research by Bryan Hamer provided useful background for the work with nest paper. This research was supported by grants to the author from the National Research Council of Canada. Reprints may be requested from S. J. Shettleworth, Department of Psychology, University of Toronto, Toronto, Ontario M5S 1A1, Canada. body with a hind leg, scent marking). The APs in the latter group were not unaffected by reinforcement, since bout length decreased over sessions with contingent food. This was interpreted as due to inhibition of these APs by the anticipation of food classically conditioned to their performance. However, other possibilities remain:

(1) The two classes of APs represent voluntary and involuntary activities. That is to say, some of the APs are intrinsically sensitive to their consequences and others are not. This is not the only factor involved, since a different grouping of activities was found with punishment (Shettleworth, 1978).

(2) The easily reinforced APs may represent a class of appetitive activities that can readily be brought under the control of a variety of positive motivational systems (Black \& Young, 1972; Hogan \& Roper, 1978). This notion suggests that different positive reinforcers would have similar effects, without predicting effects of punishment.

(3) In contrast to the first two cases, the relative modifiability of APs in frequency may be reinforcerspecific. That the activities readily increased by food reinforcement in hungry hamsters are the same as those facilitated by hunger suggests that they are the hamster's "species-specific food-seeking activities," by analogy with species-specific defense reactions to electric shock (Bolles, 1970).

(4) Response-specific factors may also be involved. For instance, grooming is depressed by both hunger and a novel environment, conditions which were present when food reinforcement was used (Shettleworth, 
1975). Grooming might not differ from the apparently more readily learned activities if it were trained under conditions generally facilitating its performance. In a similar way, Black and Young (1972) showed that rats would drink to avoid shock only when they were thirsty or the water was sweetened.

One approach to differentiating among these possibilities is to use positive reinforcers other than food for hungry animals. Such experiments can ask, first, whether the pattern of results is the same across different positive reinforcers. Second, they allow further examination of how the noncontingent effects of reinforcer presentation or deprivation are related to the contingent effects of reinforcers.

In the present experiments, two different positive reinforcers were presented to undeprived hamsters in their home cages: sunflower seeds and nest paper. Both nesting material (Jansen, Goodman, Jowaisas, \& Bunnell, 1969) and food to hoard (Richelle, Charpentier, Cornil, Brockart, \& Lalière, 1967) have been reported to reinforce barpressing in undeprived hamsters. In both cases, the animals needed access to their home cages, where they could deposit the material hoarded, in order to press at high rates.

\section{EXPERIMENT 1: EFFECTS OF NONCONTINGENT SEEDS OR PAPER}

The purpose of this experiment was to observe the undisturbed behavior of hamsters in their home cages and to determine the effects of presenting free sunflower seeds or free nest paper. Effects of presenting seeds or paper contingent on various activities could then be assessed in relation to this information. Throughout this and subsequent experiments involving presentation of seeds, the hamsters always had sunflower seeds and Purina chow in their cages as well as nest paper. Thus they were neither food deprived nor deprived of the specific reinforcers. In Experiment $1 \mathrm{a}$, the hamsters received free sunflower seeds or nothing in the testing apparatus. In Experiment 1b, the hamsters received free seeds or free nest paper.

\section{Experiment 1a}

\section{Method}

Subjects. The subjects were 12 experimentally naive golden hamsters, Mesocricetus auratus, of both sexes, raised and maintained as described by Shettleworth (1975). They were 3 to 4 months old at the start of the experiment and weighed 102 to $121 \mathrm{~g}$ (mean $112 \mathrm{~g}$ ).

Apparatus. The apparatus for delivering sunflower seeds to hamsters in their home cages is depicted in Figure 1. It included a turntable with depressions on the rim that each held one hulled sunflower seed. The turntable was behind a response panel containing a speaker, a white jewel light, a slot for a
Gerbrands rat lever, and a hole through which seeds could be presented, located as indicated in the figure. The holes for seeds and the lever were 5 and $6 \mathrm{~cm}$, respectively, from the floor of a cage when it was in place, and their centers were $11.5 \mathrm{~cm}$ apart. The hamster's home cage (dimensions 22.5 $\times 27.0 \times 22.5 \mathrm{~cm}$ ) could be placed with its door open against the response panel with lever and feeder accessible, as shown. The feeder was operated by activating a motor until the next depression or next space in the turntable was beneath the hole in the response panel. The slot for the lever was covered in this experiment.

Behavior was recorded with a keyboard wired to electromechanical programming equipment which counted the number of $1-\mathrm{sec}$ intervals in which each key was depressed. Number of bouts (i.e., number of button depressions) and total times spent were also recorded for some activities. An EsterlineAngus 20-pen recorder running at $3 \mathrm{in} .(7.62 \mathrm{~cm})$ per minute recorded the sequence of button depressions and other experimental events.

Procedure. Sessions were run during the dark phase of the light cycle in the room where the subjects lived, and had lived for at least 2 weeks before the experiment. An air-conditioner fan provided masking noise throughout. For each 20-min session, a subject's cage was placed against the feeder panel with a $25-\mathrm{W}$ red light bulb about $10 \mathrm{~cm}$ above the top of the cage. Recording began as soon as the observer could sit down.

Each subject had eight sessions, each run at the same time of day. The six animals in the seed group received free sunflower seeds on a variable-time (VT) $30-\mathrm{sec}$ schedule. The noseed group had no seeds. Whenever a seed was delivered, it was available for $10 \mathrm{sec}$ or until the hamster collected it, whichever came sooner. If the hamster was headed for the feeder near the end of $10 \mathrm{sec}$, the seed remained available for another $10 \mathrm{sec}$. During the first three sessions, a few extra seeds were delivered to the animals in the seed group to help shape them to collect. Availability of a seed was accompanied by an $800-\mathrm{Hz}, 80-\mathrm{dB}$ (General Radio Corp. Meter 1561A, Flat Scale) tone and lighting of the jewel light. When a seed was collected or at the end of its programmed availability, the turntable advanced so that a flat area between depressions was under the hole in the response panel.

All subjects were given clean cages with fresh nesting material and sawdust 3 days before their first experimental session. Thereafter, the cages were disturbed only to replace any wet sawdust and give fresh Purina chow and vegetables after observations for the day were completed. The animals had regularly had supplements of sunflower seeds prior to the experiment

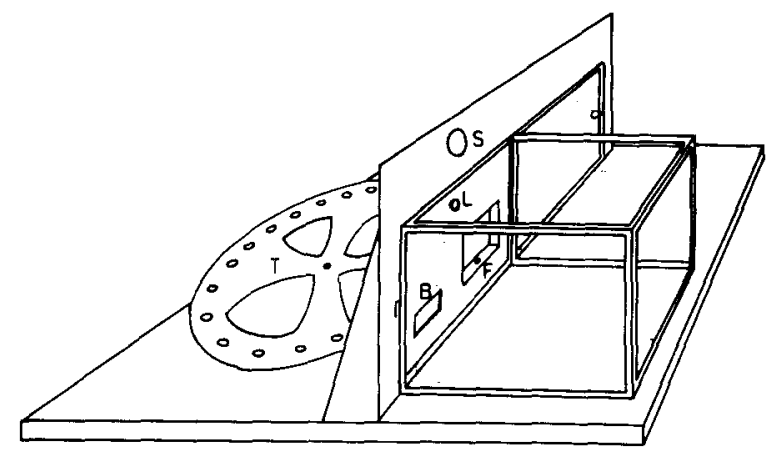

Figure 1. Apparatus for delivering sunflower seeds to hamsters in their home cages, with a cage in place. $S$, speaker; $L$, jewel light; B, slot for bar; F, feeder opening, where seeds are delivered; T, turntable. 
and had seeds in their food piles at the beginning of the experiment. Checks of the nests and food piles showed that they nearly always had some seeds during the experiment. The no-seed animals were given their regular supplements of seeds.

Behavior was recorded in the categories described in Table 1 and in several others observed too infrequently to be analyzed (see Shettleworth, 1975).

\section{Results and Discussion}

The number of $1-\mathrm{sec}$ intervals in which each AP occurred was transformed to $\sqrt{\mathrm{x}+1}$, which makes frequency data of this kind more suitable for parametric analysis (see Shettleworth, 1975). Results for each AP were subjected to analysis of variance with groups and days as the factors and $\mathrm{p}<.05$ accepted as significant. For Groups, $F(1,10)>4.96, \mathrm{p}<.05$; for Days and Conditions by Days, $\mathrm{F}(7,70)>2.17, \mathrm{p}<.05$.

The animals that received free seeds reared at the feeder panel in about 50\% of the $1-\sec$ intervals and collected most of the seeds. They generally stored these in their cheek pouches, making only one or two trips per session to their food piles to disgorge the seeds. They collected and pouched seeds in about $5 \%$ of the $1-\mathrm{sec}$ intervals and spent a similar amount of time eating. They performed these activities and panel rearing substantially and significantly more than the animals that did not receive seeds $(p<.01)$. This increase in activities related to taking the seeds was accompanied by a decrease in some other APs relative to the group that did not receive seeds. The seed group performed reliably less scratching, grooming belly and sides, shaking, manipulating nest paper, scent marking, walk-sniffing, and wall rearing than the no-seed group. There were no reliable effects on the other APs $(p>.10)$.

The activities that were suppressed when seeds were presented did not have especially high or low baselines compared to those that were unaffected such as open rearing, digging, and scrabbling, nor were they all affected in the same way by variables such as food deprivation and presentation in previous experiments (Shettleworth, 1975). Walk/sniffing and wall rearing were classified as "exploratory" activities in an open field, and wall rearing was enhanced by food deprivation. The other APs depressed by seed presentation could be classified as maintenance activities on the basis of their function. They were generally depressed in the open field and by food deprivation, but not all such APs were depressed in the seed group here. Most notably, face washing was at virtually identical levels in both groups $(\mathrm{F}<1.0)$.

\section{Method}

\section{Experiment 1b}

Subjects. The subjects were 12 naive hamsters like those in Experiment 1a, 3-5 months old and weighing $87-119 \mathrm{~g}$ (mean $101 \mathrm{~g}$ ).
Table 1

Definitions of Behavioral Categories

Action Pattern Definitions

Walk/sniff

Walk around or stand still and sniff with at least three feet touching floor.

Open rear

Stand on hind legs with both forepaws off floor and walls.

Wall rear

Stand on hind legs with at least one forepaw touching a wall.

Panel rear

Wall rear with at least one forepaw touching the dispenser panel.

Scrabble

Scrape with forepaws against a wall while standing erect. May move along wall hopping up and down while doing this. Distinguished from digging at the wall by the fact that each stroke is oriented up rather than toward the wall, and the hamster usually stretches up to its full extent. Also includes climbing, i.e., hanging from wire mesh sides of cage with all feet off the floor.

Dig

Gnaw

Scent mark

Scrape with forepaws directed in front of face at sawdust, bare floor, or wall.

Bite at wall, corner, or edge of any surface.

Press scent gland in flank against (usually) a vertical surface with arched back, raised tail, and at high intensity, distinctive minccing steps.

Wash face

Rub forepaw(s) over any part of head. Includes interspersed licking of forepaws.

Groom belly and sides Scratch and/or bite belly, sides, or legs with mouth and/or forepaws.

Scratch with hind leg

Scratch any part of body with hind foot. Includes interspersed licking of the scratching foot.

Shake

Eat

Sudden convulsive shake of whole body.

Hoard

Bite and chew food.

Pouch or depouch food or drop it onto food pile. Push food around with paws, nose, or mouth. Carry Purina Chow pellet in teeth.

Manipulate nest Hold, carry, or push nest paper with paws paper and/or mouth. Pouch or depouch nest paper.

In nest Stand or lie wholly on the nest paper.

Collect Remove seed or paper from dispenser and fit it into cheek pouches.

Chew paper

Gnaw on remains of paper sticking out of dispenser.

Apparatus. In addition to the seed delivery device, a similar one for delivering nest paper was used. The panel facing the hamsters was identical to that of the seed dispenser except that the opening for seeds was replaced with a slot through which strips of adding machine paper could be delivered by a motor and clutch system. The hamsters had to detach the paper by chewing it off. 
Procedure. The procedure was the same as that of Experiment $1 \mathrm{a}$ in all unspecified details. Animals in one of two matched groups received free seeds on VT $30-\mathrm{sec}$ just as in Experiment 1a, except that a seed remained available with the light and tone on until it was collected. The other group received $1.25 \times 2.5 \mathrm{~cm}$ strips of paper on the same schedule. The tone and light accompanied availability of paper. The paper group, like the seed group, always had nests in their cages, but to prevent too much paper from accumulating, some of it was removed at the end of each day. Each animal in the paper group had 5-10 strips of paper presented through the door of its cage on one occasion a few days before the experiment to give it practice chewing through and detaching the strips.

Free paper or seeds were given for eight sessions; then, for two sessions, the animals were placed in extinction and no seeds, paper, tones, or lights were given. The data were analyzed as in Experiment 1a.

\section{Results}

The hamsters collected significantly more free seeds than free paper. The group receiving seeds collected a mean of at least 35 of the $40 \pm 1$ available seeds in all of the last four sessions. Individuals often collected all the possible seeds in a session. In contrast, 35 was the most strips of paper a hamster ever collected in a session and the group means never exceeded 30.

These data might be taken to mean that under the conditions of this experiment paper is a weaker stimulus than seeds, or the animals are less motivated to obtain it. Be that as it may (see Hogan \& Roper, 1978, for a discussion of the problems of comparing strength of different reinforcers), the hamsters in the two groups behaved in qualitatively different ways. The hamsters receiving free seeds reared at the feeder panel, "waiting for seeds," in as many as $2 / 3$ of the available 1 -sec intervals in the final sessions. They also gnawed at and dug around the opening for seeds. Digging and panel rearing were both significantly higher in the seed group, and gnawing was also higher, though not significantly so $(\mathrm{p}<.10)$.

The animals receiving paper tended to leave the panel after collecting paper. They panel reared in only $25 \%-30 \%$ of the $1-\mathrm{sec}$ intervals. Despite the fact that they could and did store many strips of paper in their cheek pouches after collecting them, they frequently returned to their nests and spent about $10 \%$ of the 1 -sec intervals manipulating both the paper they had collected and that in their existing nests. This was significantly more than the $0 \%-2 \%$ spent by the seed group. Time sitting in the nest was also significantly higher in the paper group.

The paper animals also spent more time walk/sniffing and wall rearing than the seed group, though the latter difference was only marginally significant $(p<.06)$. The paper animals also open reared reliably more, reflecting the observation that, on the frequent occasions when they were not at the panel, as a strip of paper appeared they open reared to the tone and light before approaching the paper.

Although the paper group collected fewer reinforcers than the seed group, they actually spent more time in the act of collecting (i.e., chewing off and pouching the paper, as compared to picking up and pouching seeds). Thus, increases in the various APs mentioned above are not due in a direct way to the smaller number of reinforcers collected.

The APs not mentioned above, including scrabbling and face washing, were performed at similar (not reliably different) levels in the two groups. The overall levels of the various APs were generally similar in the seed groups in this experiment and Experiment 1a. The differences between the groups disappeared in extinction, although the paper group continued to manipulate nest paper more $\mathrm{F}(1,10)>4.96, \mathrm{p}<.05$.

\section{Discussion}

The following conclusions can be drawn from the two parts of Experiment 1. First, under the conditions of this experiment, intermittent free nest paper seems to exert weaker control over the hamsters' behavior than intermittent free sunflower seeds. This difference may be confined to intermittent presentation. Roper (1975), using mice, and Jansen et al. (1969), with hamsters, noted that when nesting material was presented at a high rate the animals collected it rapidly but when it was intermittent they appeared to lose interest. In both cases, when delivery was intermittent the animals began spending more time with the paper they had already collected. Hogan and Roper (1978), reviewing the literature on nest material as a reinforcer, suggest that, in rodents that are neither maternal nor cold, motivation for nesting material is induced by external factors and performance for intermittent paper follows from this. This is not an adequate account of the differences between seeds and paper in Experiment $1 \mathrm{~b}$ since it would seem that motivation to obtain seeds was also induced by external factors (i.e., the availability of seeds) inasmuch as the animals were deprived of neither food nor seeds.

A second conclusion relevant to the use of seeds and paper as reinforcers for different APs is that, although they do have predictably different effects on the APs involving contact with them, such as manipulating nest paper, they otherwise do not have much differential nonassociative effect on behavior. What differences there are, such as the increased walk/sniffing and open rearing in the paper group in Experiment $1 \mathrm{~b}$, are concomitants of the different modes of collecting the reinforcer-i.e., waiting at the feeder for seeds vs. walking to the nest and back with free paper. The results of the two parts of the experiment taken together indicate that the time spent with either reinforcer is "compensated for" by depression of only some other activities, such as certain maintenance activities. 


\section{EXPERIMENT 2: SEED REINFORCEMENT FOR THREE BEHAVIORS}

In this experiment hamsters were reinforced with sunflower seeds for open rearing, scrabbling, or face washing. When hungry hamsters were reinforced with food for performing these APs in an open field (Shettleworth, 1973, 1975), open rearing and scrabbling rapidly increased to high rates while face washing did not. However, scrabbling and face washing showed bigger effects of punishment than open rearing (Shettleworth, 1978), indicating that the differences with food reflect some reinforcer-specific factors and not just intrinsic modifiability of the APs.

Face washing was of particular interest here since it is depressed by food deprivation and by a novel environment (Shettleworth, 1975), conditions which prevailed in the previous studies. Since the hamsters seemed to learn the association between face washing and food in these studies, perhaps the failure of face washing to be performed at a high rate was due to these inhibitory factors. Black and Young (1972) showed that rats could learn to drink water to avoid shock but they would only do so at a high rate if they were water deprived or the water was sweetened, i.e., if conditions facilitating drinking were present. If a similar mechanism applies to face washing in hamsters, reinforcing nondeprived hamsters with seeds for face washing in their home cages should result in substantial amounts of face washing.

\section{Method}

Subjects and Apparatus. The subjects were 12 experimentally naive hamsters like those in Experiment 1, 21/2 to 4 months old at the start of the experiment and weighing 89 to $136 \mathrm{~g}$ (mean $113 \mathrm{~g}$ ). The apparatus was that used in Experiment 1a.

Procedure. The general procedure was that of Experiment 1a. For the first three sessions, all subjects were magazine trained with free seeds on VT 30 sec and a few extras as necessary to shape them to approach the feeder when the tone and light came on.

After the second session of magazine training, whether each subject was to be reinforced for open rearing, scrabbling, or face washing was determined according to the following criteria, in order of importance. First, the total time for which the to-bereinforced AP had been performed during the first two sessions had to be about the mean group level for that AP during magazine training here and in Experiment 1 . Second, two animals from each group were included in each of the two runs necessary to complete the experiment. Third, each group had to be about equally magazine trained, as measured by the number of reinforcements collected in the first two sessions and the mean latency to collect them. Finally, where other criteria permitted, the two sexes and the litters used were equally represented in all groups.

After a third session of magazine training, reinforcement began for the AP chosen. Pressing the key indicating that AP was being performed started a train of $10 / \mathrm{sec}$ pulses which counted down a predetermining counter set to deliver reinforcement and reset when it had received 10 pulses. It did not reset at the end of a bout unless the last pulse received during a bout was the 10th, and it could reset and deliver a seed at any time during a bout. Thus the hamster was reinforced each time it had spent approximately 1 sec performing the selected AP, regardless of the number of bouts during which this time was accumulated. This reinforcement procedure will be referred to as a fixed cumulative duration (FCD) $1-\sec$ schedule of reinforcement.

Nine sessions of reinforcement followed the three of magazine training. During these sessions, each hamster progressed independently through the following sequence of increasingly demanding CD schedules: FCD 1, FCD 2, VCD 3, VCD 4, and VCD 5. (For variable cumulative duration-VCD-schedules, the pulse train was gated through a probability generator.) A subject advanced to the next schedule in the sequence whenever it earned at least 50 reinforcements in one session on a given schedule, except that two such sessions were required before VCD 5 sec. This procedure insured that a ceiling was not imposed on performance by satiation or by frequent pauses to collect and depouch seeds. Reinforcement availability was signaled and, except for its contingency on a particular AP, programmed as during magazine training. Two sessions of extinction followed the nine sessions of reinforcement. The tone and light were never presented during extinction, nor was the turntable operated.

Beginning in the third session of magazine training, a cumulative record, bout length distribution (lengths of bouts in 10ths of seconds), and total time spent performing the selected AP were recorded for each animal on all remaining sessions. All behavior was recorded throughout all sessions. Once the rate of the reinforced response had begun to increase toward 100 bouts or more per session, only every second bout was included in the bout length distributions, since the apparatus did not produce accurate records if every bout was included.

\section{Results and Discussion}

The mean number of seconds per 1,200-sec session that each group performed its reinforced AP is shown in Figure 2. Just as with food reinforcement for hungry hamsters (Shettleworth, 1975), open rearing and scrabbling showed large and immediate increases in rate while face washing increased only slightly and gradually. The group curves for open rearing and scrabbling were typical of individuals in those groups. There was no overlap between the open-rearing and face-washing groups after the first session with contingent seeds. The scrabbling subjects were generally like those reinforced for open rearing, except that one of them hardly scrabbled at all for the first four sessions after magazine training. It then scrabbled at a high rate for two sessions, but without collecting many seeds. For the next two sessions, it scrabbled and collected seeds, but at the end of training it appeared to lose interest in seeds and spent most of the remaining sessions sleeping and grooming in its nest. No other animals in this or Experiment 1 behaved in this way.

The data for magazine training displayed in Figure 2 indicate that differences in the groups' terminal performances were not correlated with the initial levels of the APs. Face washing and scrabbling had similar initial rates, while open rearing was higher, but reinforcement affected scrabbling and open rearing similarly. The results displayed in Figure 2 are also not due to some feature of the reinforcement procedure as such. Each of the three reinforced APs either remained at a 


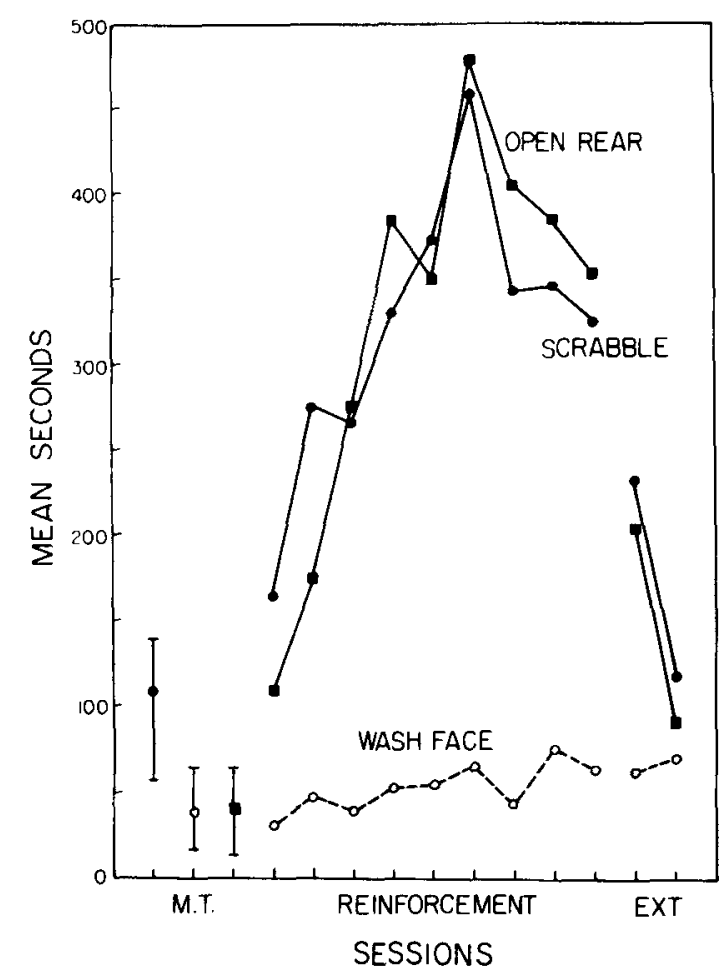

Figure 2. Mean total seconds per 1,200-sec session spent on the reinforced AP by hamsters reinforeed with sunflower seeds for open rearing, scrabbling, or face washing in Experiment 2. Data for magazine training (MT) are means and ranges for the last session. EXT, extinction.

fairly constant low level or decreased over sessions when another one of the APs was reinforced.

The number of bouts differed among the APs in a similar way to total times: face-washing bouts increased gradually, while open-rearing and scrabbling bouts increased sharply with the onset of contingent seeds. The mean number of bouts per session of scrabbling and open rearing later declined, reflecting the lengthening bouts of these APs as described below.

Face washing differed from open rearing and scrabbling in that its bout length steadily decreased over sessions with reinforcement (Figure 3). That of open rearing steadily increased, while that of scrabbling, which began relatively high, first decreased and then increased. To some extent, the increasing bout lengths of open rearing and scrabbling can be attributed to the fact that all subjects in these groups except the anomalous scrabbling subject, whose data are omitted from Figures 3 and 4, were reinforced for ever-increasing average durations of the given APs. All these animals moved beyond the initial schedule by the fifth session of contingent seeds at the latest. Thus, the longer their bouts, i.e., the less they interrupted performance of the reinforced AP with other activities, the sooner they were reinforced. Similarly, the initial decrease in the bout length of scrabbling can be accounted for by the fact that scrabbling was interrupted by delivery of a seed every $1 \mathrm{sec}$. The gradually decreasing bout length of face washing can be accounted for in a similar way, since all but one subject in this group remained on the FCD 1-sec schedule for all nine sessions with contingent seeds.

The relatively long bouts of face washing at the beginning of training were reflected in long latencies to collect seeds once they were made available (Figure 4). While, on the first day with contingent seeds, the open rearing and scrabbling subjects had collection latencies similar to those at the end of magazine training, the face-washing subjects' scores all increased greatly, except for the animal that showed the most sign of learning, and in subsequent sessions latencies decreased slowly. The two face-washing animals with the longest collection latencies also had the longest bout lengths in that group. Together, the gradually decreasing bout lengths and collection latencies suggest that the hamsters reinforced for face washing had to learn to interrupt face washing to collect seeds. The scrabbling and open rearing animals interrupted these APs to collect even at the outset. Their speed of collecting did improve later, largely due to their acquiring stereotyped ways of performing the reinforced AP near the feeder.

One implication of the data shown in Figure 4 is that initially there was a longer delay of reinforcement for face washing than for the other APs. This could account for its slow rate increase. The face-

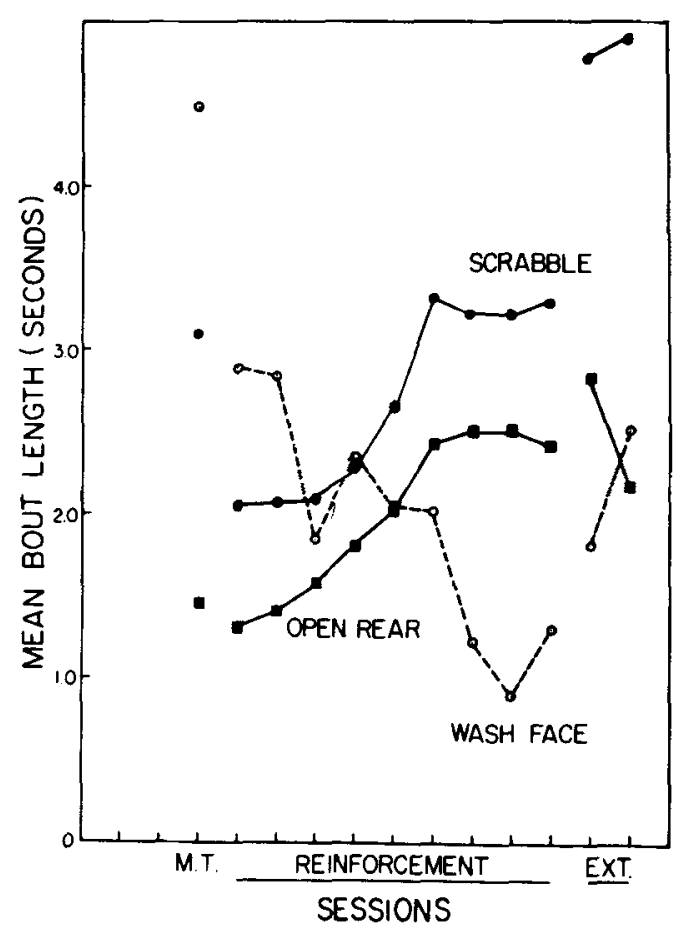

Figure 3. Mean bout lengths of the reinforced APs in Experiment 2. 


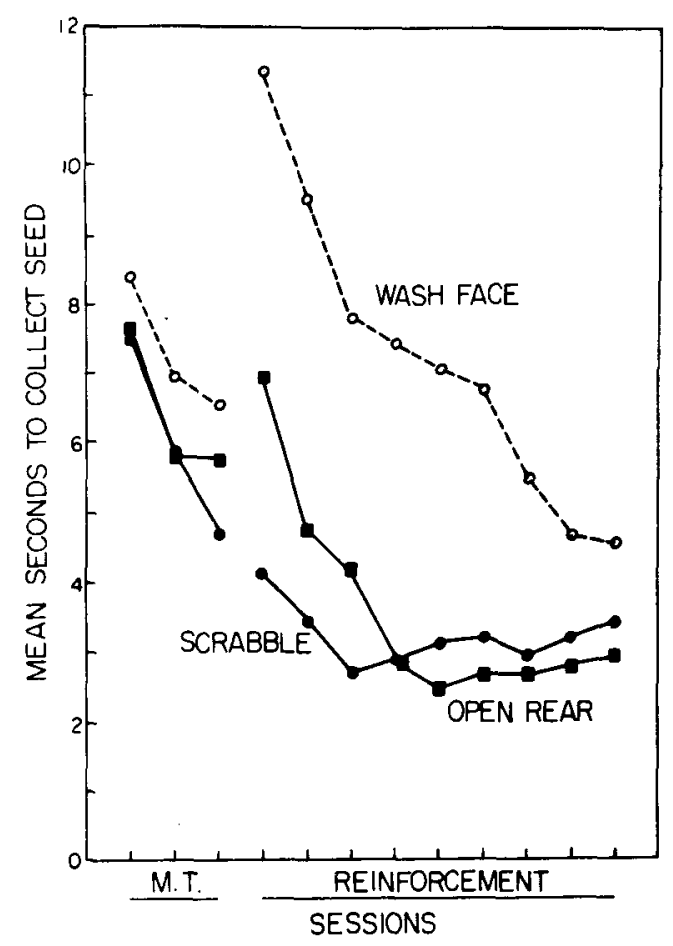

Figure 4. Mean latencies to collect seeds after they were presented to the three groups of hamsters in Experiment 2.

washing group's collection latencies did eventually become similar to those of the other two groups at the beginning of training, but without face washing showed any marked rate increase. Moreover, two features of their behavior showed that the face-washing animals were doing more than just learning to pause in face washing each time a seed was delivered. First, although the mean bout length of face washing came to be about the $1 \mathrm{sec}$ required to earn a seed, most animals came to perform a large number of bouts shorter than this while standing near the feeder. In addition, they performed some "normal" grooming in their nests, scratching, and grooming belly and sides, which they seldom interrupted to collect seeds. The appearance of sequences of very short bouts, several of which were necessary to earn a reinforcer, could clearly be seen on the cumulative records.

Further evidence that the animals were not just interrupting face washing for seeds is seen in the bout length data for extinction (Figure 3). All subjects reinforced for face washing had shorter bouts on the first day of extinction than on the last day of magazine training or the first day of contingent seeds. Thus it would appear that sunflower seeds were associated with face washing even though this association did not result in a large amount of time face washing and the accumulation of a large number of seeds. The results with open rearing and scrabbling show that such performance is by no means a necessary result of any aspect of the procedure other than the particular $\mathrm{AP}$ reinforced. Hamsters quickly learned to perform these APs almost continuously and hoarded large numbers of sunflower seeds. Even with the steadily advancing response requirement, animals reinforced for scrabbling or open rearing routinely collected $90-100$ or more seeds per session. Moreover, at the beginning of training they collected sunflower seeds more promptly than the animals receiving seeds contingent on face washing.

The previously reported study (Shettleworth, 1975) using food as a reinforcer for open rearing, scrabbling, face washing, and other APs in hungry hamsters in an open field revealed the same sort of differences in bout lengths and collection latencies found here. In that case, the response requirement was $2 \mathrm{sec}$ of the reinforced AP rather than $1 \mathrm{sec}$. Hamsters reinforced for face washing began to perform a preponderance of bouts much shorter than $2 \mathrm{sec}$, although initially most bouts were relatively long. It was argued in that case that the hamsters had associated face washing with food, but the anticipation of food classically conditioned to the initiation of face washing inhibited its further performance. A similar mechanism appears to be operating here. In both cases, it is not clear whether this is the sole reason why face washing is performed so rarely when it is reinforced. Other factors, such as face washing being relatively difficult to associate with food, may also be involved.

\section{EXPERIMENT 3: PAPER REINFORCEMENT FOR THREE BEHAVIORS}

In this experiment, hamsters were reinforced with nest paper for open rearing, scrabbling, or face washing. In Experiment 3a, all three APs were reinforced in a procedure paralleling that of Experiment 2. In Experiment $3 b$, face washing was reinforced under special conditions designed to facilitate training.

\section{Method}

\section{Experiment 3a}

Subjects. The subjects were 12 golden hamsters like those in Experiment 1, 4-6 months old and weighing 92-123 g (mean $105 \mathrm{~g}$ ). A 13 th subject was discarded for failing to collect paper in magazine training.

Apparatus. The apparatus described in Experiment $1 \mathrm{~b}$ for delivering paper strips was employed.

Procedure. The procedure was the same as in Experiment 2 except that the reinforcer was a $1.25 \times 2.5 \mathrm{~cm}$ strip of paper. Each strip remained available, with the tone and light on, until the hamster collected it. Subjects had preliminary practice cutting paper, as in Experiment 1b. Two subjects, from two different groups, each had 1 extra day of magazine training because they were slow to begin collecting paper. Data for their 1 st day are omitted.

\section{Results and Discussion}

Contingent nest paper increased the time spent scrabbling or open rearing but had essentially no effect on 
face washing (Figure 5). All the animals reinforced for open rearing or scrabbling showed large sustained increases over baseline, while the rate of reinforced face washing always remained low and variable.

The maximum times spent on reinforced scrabbling and open rearing were slightly lower than when these APs were reinforced with seeds (cf. Figure 2). This could partly be due to the longer time necessary to collect paper. The animals in these groups generally collected means of 50 or more strips of paper per session; individuals sometimes collected more than 100. In contrast, the mean number of strips collected by the face-washing animals was always less than 5 , even though, like the other two groups, these animals had been collecting 20-30 strips per session at the end of magazine training.

None of the three APs was increased when another AP was reinforced. Open rearing decreased; scrabbling and face washing remained constant. The mean level of scrabbling increased during extinction of open rearing, just as it did in the group formerly reinforced for scrabbling. However, the nature of scrabbling changed during extinction in the latter group. Instead of scrabbling at the wall near the paper dispenser, the animals began to spend long periods hanging from the walls and ceiling of the cage (i.e., climbing, which was counted as scrabbling).

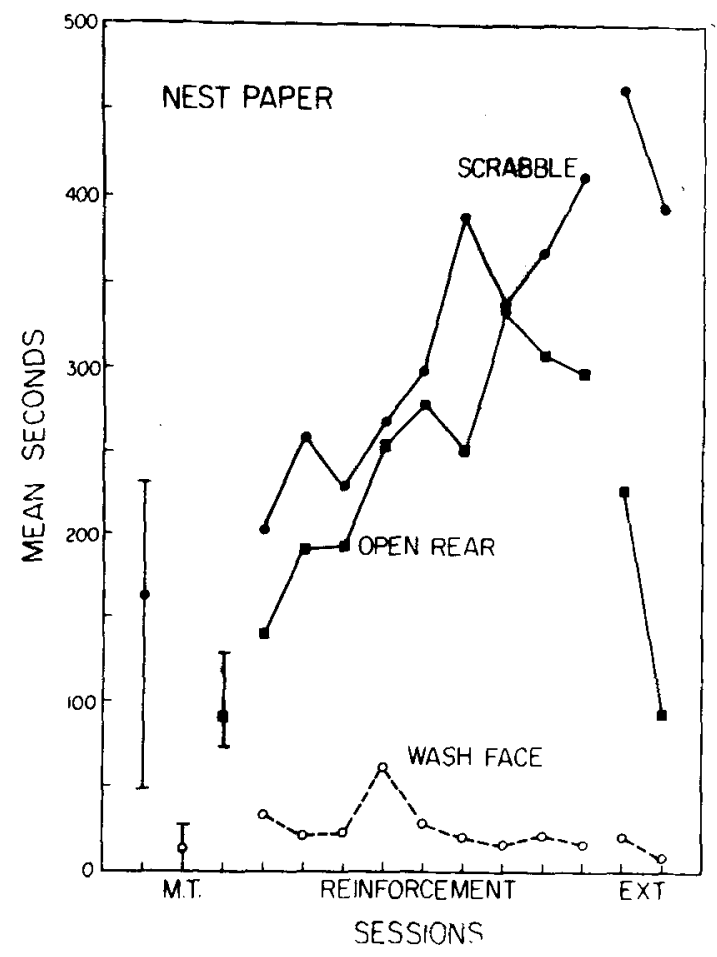

Figure 5. Mean total seconds per 1,200 sec spent on the reinforced AP by hamsters reinforced with nest paper in Experiment 3a. Data for magazine training (M.T.) are means and ranges for the last session.

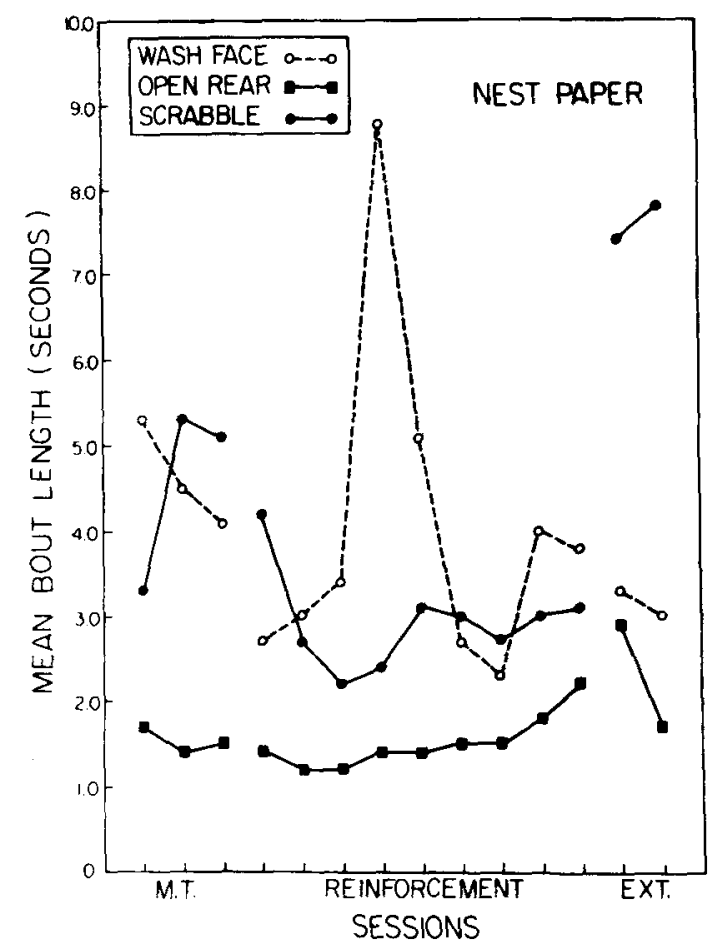

Figure 6. Mean bout lengths of the reinforced APs in Experiment 3a.

Not only the overall amount of face washing, but also its bout lengths, failed to change with reinforcement. The results for each scrabbling and open-rearing subject parallel the mean curves in Figure 6 and were like those with seeds. The scrabbling bout length decreased at first and then increased, while that of open rearing increased throughout training. The bout lengths of face washing, however, showed no systematic trend. No individual showed the steadily decreasing average bout length and the very short bouts found with seeds and food.

Collection latencies also differed among the groups. In the open-rearing and scrabbling groups, collection latencies quickly became less than in magazine training while those of the face-washing group increased and remained high, with the paper often not detached until some time after the bout of face washing had ended.

In summary, the results with scrabbling and open rearing show that hamsters will perform some APs at high rates to obtain nest paper under the present conditions. Not only did hamsters reinforced for face washing fail to increase face washing, but also their bout lengths and collection latencies gave no indication that they had associated face washing with paper.

One reason for the apparent failure to learn may have been the low operant level. Obviously, the hamsters had relatively little opportunity to associate face washing with paper. The low operant level could also decrease motivation for paper and thus make the hamsters less likely to collect it when it did come, since 
it will be recalled that Experiment 1 and data from other rodents (Hogan \& Roper, 1978) suggest that presentation of paper itself primes motivation for paper. With a response that had a high operant level or was quickly associated with its consequences, a positive feedback loop would exist, but the opposite would be the case with a response with a low operant level or with one that was slow to be associated with given consequences.

In addition, Roper (1975) showed that mice were especially sensitive to operant-reinforcer distance with nest paper as compared to food reinforcement. The hamsters' face washing in their home cages was most often performed in the nest, which was invariably at the side of the cage farthest from the door and dispenser. Scrabbling and open rearing, in contrast, were likely to be performed outside the nest (see Shettleworth, 1975, Experiment 1) necessarily nearer the dispenser. Thus the failure of face washing to be affected by nest paper reinforcement in this experiment might be due to any several generalized variables-large operant-reinforcer distance, low operant level, and low motivation for infrequently presented nest paperand not reflect a low associability of face washing with paper.

\section{Experiment 3b}

This experiment included three manipulations to increase the likelihood that face washing would be affected by contingent nest paper. First, an attempt was made to make the hamsters respond more quickly to nest paper presented after face washing by increasing the amount of magazine training. Second, the number of sessions with paper contingent on face washing was increased. Third, the operant level of face washing was increased by spraying the animals with a fine mist of water. Konorski (1967) reviewed experiments in which a similar procedure was used while training dogs and cats to perform grooming movements for food. In that work, the desired movement was at first elicited with the appropriate stimulus, but after a certain amount of training the movement was performed without the elicitor. An analogous test was performed late in the present experiment by spraying and withholding spraying on alternate sessions.

\section{Method}

Subjects. The subjects were five 5- to 7-month-old hamsters like those in Experiment 1, weighing $111-121 \mathrm{~g}$ (mean $116 \mathrm{~g}$ ).

Apparatus and Procedure. The procedure was like that for the face-washing group in Experiment 3a except that there were 6 sessions of magazine training and 14 of contingent paper, followed by two sessions of extinction (one subject had no extinction sessions). On Sessions 5-15, 17, 19, 21, and 22 , the animals were sprayed with a fine mist of water from a house-plant sprayer at the beginning of the session and every $5 \mathrm{~min}$ thereafter. The sprayer was held about $20 \mathrm{~cm}$ from the cage and the lever fully depressed three times. This covered the part of the animal's fur exposed to the spray with small drops of water. The recording apparatus was off while the animals were being sprayed.

\section{Results and Discussion}

Spraying the hamsters with water greatly increased the time they spent face washing (Figure 7) and engaging in other types of grooming. With spraying, the operant levels of face washing were well within the ranges of operant levels for scrabbling and open rearing in Experiment 3a (Figure 5). Nevertheless, the overall time the animals spent face washing was clearly not increased by contingent nest paper during spraying. Unlike the cases described by Konorski (1967), reinforced face washing tended to decrease each time spraying was withheld. Out of 25 transitions from spraying to not spraying, or the reverse, 20 were in the direction of more face washing with spraying.

Examination of the individual data for bout length, number of reinforcements and reinforcement collection latencies revealed that two of the subjects came to have short $(<10 \mathrm{sec})$ collection latencies and short bout lengths $(<2 \mathrm{sec})$, and to collect relatively large numbers of reinforcements. Thus they behaved similarly to the animals reinforced with seeds for face washing in Experiment 2. Even they, however, did most of their face washing and paper collecting right after being sprayed. The other three subjects varied around their initial levels throughout. Therefore it cannot be concluded that nest paper does not reinforce face washing in hamsters. The conditions of this experiment are apparently marginal for its doing so. The strength of nest paper as a reinforcer might be further increased by making the animals cold, or pregnant, or depriving them of nests (Hogan \& Roper, 1978).

\section{GENERAL DISCUSSION}

When undeprived hamsters were reinforced with sunflower seeds or nest paper in their home cages for open rearing, scrabbling, or face washing, the pattern of results obtained in the present experiments was the same as that reported previously (Shettleworth, 1973,

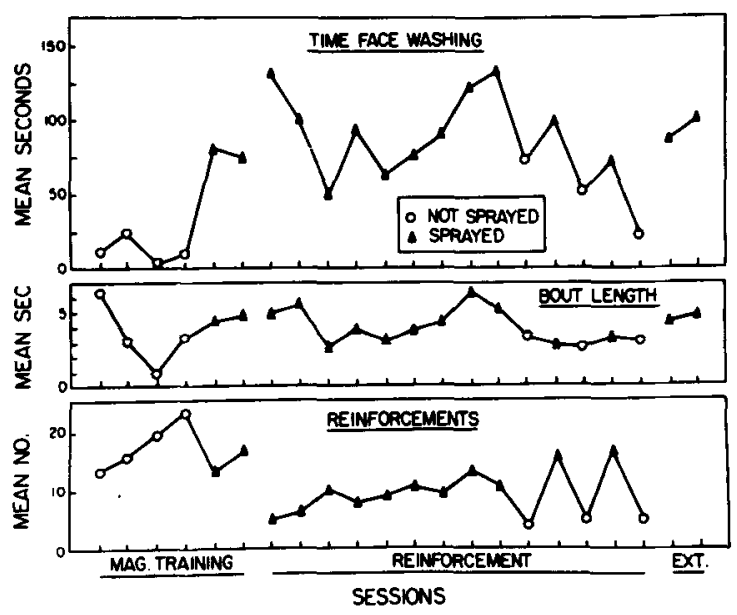

Figure 7. Mean time face washing, bout length, and reinforcements collected in Experiment 3b. 
1975) when food reinforcement was given to deprived hamsters in an open field for the same APs. Open rearing and scrabbling showed large and immediate rate increases, while face washing showed little or none. Moreover, reinforced scrabbling and open rearing gradually increased in bout length and the hamsters' latencies to collect reinforcers became quite short. In contrast, the bout length of face washing became, if anything, shorter during reinforcement and the collection latencies decreased slowly, if at all. Thus, the results found previously with food for hungry hamsters are general to two other positive reinforcers, at least for open rearing, scrabbling, and face washing.

Experiment 1 showed that APs not directly involved in collecting paper or seeds were not differentially affected by noncontingent presentation of these stimuli. Experiments 2 and 3 showed that contingent paper and seeds also do not have differential effects, except that, overall, paper had weaker control over the hamsters' behavior than seeds. In the food reinforcement experiments, the APs that showed large and immediate effects of contingent presentation were facilitated relative to baseline levels by food deprivation, while those that showed only little increase in rate like face washing were suppressed. With free food, the APs in the former group (e.g., open rearing and scrabbling) decreased and were replaced by large amounts of panel rearing, while those in the latter group (e.g., face washing) were unaffected. However, open rearing, scrabbling, and face washing were unaffected by free seed presentation in Experiment 1a of the present studies, and in Experiment $1 \mathrm{~b}$ free seeds and free paper had differential effects only on open rearing. Thus, differences between open rearing and scrabbling, on the one hand, and face washing, on the other, during reinforcement are obtained without the animal being in a state that differentially affects the two classes of APs.

Related to this point is the finding that face washing still shows little or no facilitation by reinforcement even under conditions favoring its performance-i.e., with the animals in their home cages and not food deprived (cf. Shettleworth, 1975) and sprayed with water. Various comparisons within and between Experiments 2 and 3, together with the results with food reinforcement (Shettleworth, 1975) suggest that two distinct processes are involved in the effects of paper, seed, or food reinforcement on face washing. First, face washing is more difficult to interrupt than scrabbling or open rearing with these conventionally presented reinforcers. In both Experiment 2 and Experiment 3, the hamsters' latencies to collect reinforcers at the beginning of contingency training were longer with face washing than with the other APs. The fact that the number of free reinforcers collected decreased when grooming was elicited with spraying (Figure 7) also suggests that face washing is motivationally incompatible with collecting paper, seeds, or food. With seeds or food at least, and sometimes with nest paper, the animals do learn to interrupt face washing and collect, as shown by the concomitant decreases in bout lengths and collection latencies late in training. But this is not all they are learning, for bout lengths become shorter than necessary to earn the reinforcer. This was especially obvious with food reinforcement, but it also appeared to some extent with seeds in Experiment 2. Moreover, even when the animals have obviously learned that face washing is followed by a reinforcer, and collect it as quickly as animals reinforced for scrabbling or open rearing do at the beginning of training, they still do not face wash as often as possible, since the response time and number of reinforcers earned remain low.

To return to the alternatives mentioned in the introduction, this kind of performance suggests that grooming is, indeed, "involuntary," as has been assumed by others (Hearst, 1975; Shettleworth, in press). However, this term is little more than a label for the finding that it shows unconventional effects of several positive reinforcers in several species. It does not provide a detailed account of the special properties of grooming compared to other APs during positive reinforcement and punishment in hamsters or other species.

\section{REFERENCES}

Black, A. H., \& Young, G. A. Constraints on the operant conditioning of drinking. In R. M. Gilbert \& J. R. Millenson (Eds.), Reinforcement: Behavioral analyses. New York: Academic Press, 1972. Pp. 35-50.

Boltes, R. C. Species-specific defense reactions and avoidance learning. Psychological Review, 1970, 77, 32-48.

Hearst, E. The classical-instrumental distinction: Reflexes, voluntary behavior, and categories of association learning. In W. K. Estes (Ed.), Handbook of learning and cognitive processes (Vol. 2). Hillsdale, N.J: Erlbaum, 1975.

Hogan, J. A., \& Roper, T. J. A comparison of the properties of different reinforcers. Advances in the Study of Behavior, $1978,8,155-255$.

Jansen, P. E., Goodman, E. D., Jowaisas, D., \& Bunnell, B. N. Paper as a positive reinforcer for acquisition of a bar press response by the golden hamster. Psychonomic Science, $1969,16,113-114$.

KoNORSKI, J. Integrative activity of the brain. Chicago: University of Chicago Press, 1967.

Richelle, M., Charpentier, C., Cornil, E., Bronckart, J. P., \& LALIÈre, C. L'amassement comme motivation dans le conditionnement du hamster. Psychologie Belgique, 1967, 7, 67-74.

ROPER, T. J. Paper as a reinforcer for fixed-ratio responding in mice. Learning and Motivation, 1975, 6, 327-343.

Seligman, M. E. P. On the generality of the laws of learning. Psychological Review, 1970, 77, 406-418.

ShetTlew ORTH, S. J. Constraints on learning. Advances in the Study of Behavior, 1972, 4, 1.68.

ShetTleworth, S. J. Food reinforcement and the organization of behavior in golden hamsters. In R. A. Hinde \& J. Stevenson-Hinde (Eds.), Constraints on learning: Limitations and predispositions. London: Academic Press, 1973. Pp. 243-263.

ShetTLEWORTH, S. J. Reinforcement and the organization of 
behavior in golden hamsters: Hunger. environment, and food reinforcement. Journal of Experimental Psychology: Animal Behavior Processes, 1975, 1, 56-87.

ShETTLEW ORTH, S. J. Reinforcement and the organization of behavior in golden hamsters: Punishment of three action patterns. Learning and Motivation, 1978, 9, 99-123.

ShetTlew ORTH, S. J. Constraints on conditioning in the writings of Konorski. In A. Dickinson \& R. A. Boakes (Eds.), Mechanisms of learning and motivation: Proceedings of $a$
Memorial Conference for Jerzy Konorski. Hillsdale, N.J: Erlbaum, in press.

Stevenson-Hinde, J. Constraints on reinforcement. In R. A. Hinde \& J. Stevenson-Hinde (Eds.), Constraints on learning: Limitations and predispositions. London: Academic Press, 1973. Pp. 285-296.

(Received for publication September 22, 1977; revision accepted December $29,1977$. ) 